\title{
Q-switched tunable solid-state laser using a MOEMS mirror
}

\author{
Alan Paterson, Ralf Bauer, Walter Lubeigt, Deepak Uttamchandani \\ University of Strathclyde, Centre for Microsystems and Photonics, Glasgow, UK
}

\begin{abstract}
Simultaneous wavelength tuning and Qswitching of a Yb:KGW laser using a single, electrothermally actuated MOEMS mirror is reported for the first time. A $\mathbf{1 5 . 4} \mathbf{~ n m}$ tuning range is achieved at $2.06 \mathrm{kHz}$ pulse repetition frequency.
\end{abstract}

Keywords-MOEMS, micromirror, electrothermal actuation, solid-state laser, tunable laser, $Q$-switch

\section{INTRODUCTION}

Using MOEMS as alternatives to bulk optics in laser systems offers innovative and cost-effective routes for miniaturization and functional flexibility. In the past decade, their use in solid-state lasers as active Q-switch devices has been introduced [1-5]. Whilst the MOEMS function as laser mirrors, rapid scanning through laser alignment enables short laser pulses without the requirement of electro-optic or acoustooptic modulators. The use of MOEMS is already well established for wavelength tuning of semiconductor lasers [6]. In relation to bulk solid-state lasers, the use of an electrothermally actuated micromirror was recently reported for wavelength tuning [7].

In this paper, we present the first demonstration of a novel, multi-function solid-state laser enabled by multi-axis MOEMS actuation. A gold-coated micromirror with 2D resonant and/or stepped tilt control is used, where electrothermal actuation on orthogonal axes enables simultaneous Q-switching and wavelength tuning of an end-pumped $\mathrm{Yb}: \mathrm{KGW}$ laser. The demonstration provides a platform for further development to exploit the synergies between MOEMS and solid-state laser technology.

\section{MOEMS DESIGN \& CHARACTERIZATION}

A commercial silicon-on-insulator multi-user process offered by MEMSCAP Inc. was used for MOEMS fabrication. The single crystal silicon device layer has a thickness of $10 \mu \mathrm{m}$ and is released from a silicon substrate through a deep reactive-ion etch and a preceding wet etch for the thin oxide layer between. The circular micromirror surface, shown in Figure 1, has a diameter of $1.4 \mathrm{~mm}$, a concave radius of curvature (ROC) of $50 \mathrm{~mm}$, and is coated with a $200 \mathrm{~nm}$ layer of gold for $96 \%$ reflectance in the near infrared. Four radially positioned actuators with $1800 \mu \mathrm{m}$ long, $40 \mu \mathrm{m}$ wide beams enable $2 \mathrm{D}$ control of the micromirror tilt. Actuation is achieved by flowing current through the outermost beams of an This work was supported by the EPSRC under grant EP/L505080/1.

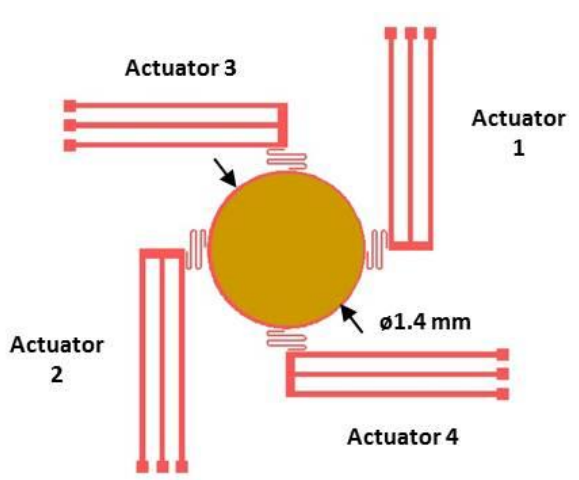

Figure 1: Schematic of the electrothermally actuated micromirror with four labelled actuators

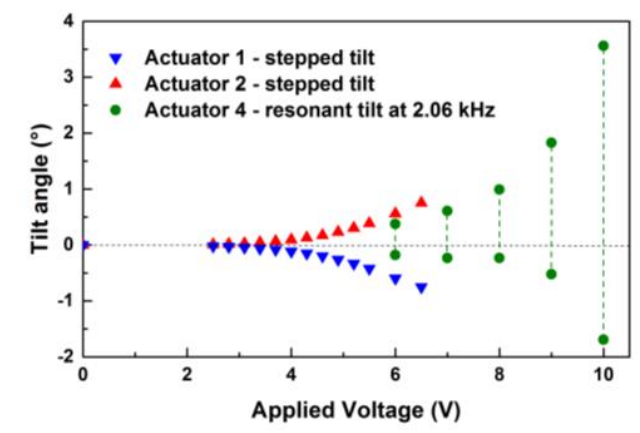

Figure 2: Plot of micromirror tilt angle against independently applied actuator voltage

actuator, resulting in Joule heating and thermal expansion. The open circuit central beam acts as a mechanical constraint to force an out-of-plane movement and tilt the micromirror. Stepped tilt can be achieved through applying a DC voltage signal, whereas scanning tilt can be achieved through applying an $\mathrm{AC}$ voltage signal at a resonant frequency of the micromirror.

The experimentally measured relationship between actuator voltage and micromirror tilt angle is shown in Figure 2. Stepped tilt was instigated at approximately $2 \mathrm{~V}$ and rose exponentially towards $0.75^{\circ}$ at $6.5 \mathrm{~V}$ for actuators 1 and 2. Resonant scanning occurred through control of actuator 4 at $2.03 \mathrm{kHz}$. Coupling between resonant modes, arising from identical orthogonal actuators, resulted in an elliptical scan. The device was therefore driven slightly off-resonance, at $2.06 \mathrm{kHz}$, to exhibit a less elliptical scan. A micromirror scan angle of $5.3^{\circ}$ was achieved by applying a $10 \mathrm{~V}_{\mathrm{pp}}$ signal with a frequency of $2.06 \mathrm{kHz}$ to actuator 4. The resonant scan is offset by 


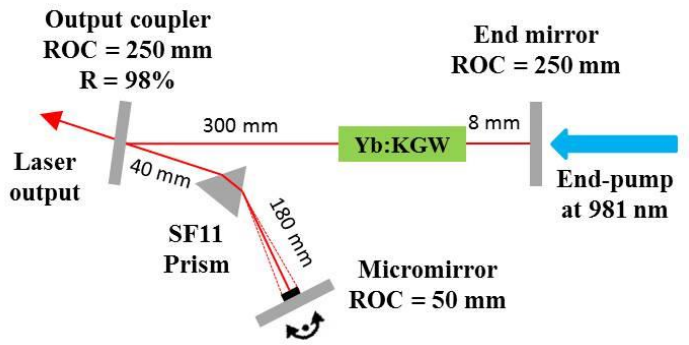

Figure 3: Schematic of the laser setup incorporating the micromirror

approximately $25 \%$ of the total scan angle towards the rising direction of the actuator. The orthogonal stepped and resonant tilts remained stable under simultaneous actuation with less than $5 \%$ variation in tilt angle compared to that achieved from independent actuation.

\section{LASER SETUP AND RESULTS}

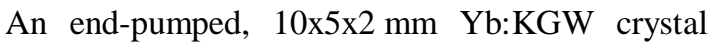
was used as the laser gain medium to facilitate a theoretical emission band from $\lambda=1020 \mathrm{~nm}$ to $1060 \mathrm{~nm}$. The resonator, shown in Figure 3, consists of a high-reflectance end mirror, a $98 \%$ reflectance output coupler, an equilateral dispersing prism and the micromirror. The end mirror and output coupler both have a ROC of $250 \mathrm{~mm}$, with the output coupler focusing the reflected light onto the micromirror. The SF11 dispersing prism of $20 \mathrm{~mm}$ base length is angled at $61^{\circ}$ to the incident laser light, corresponding to the minimum angle of deviation of the light through the prism. The stepped tilt of the micromirror from actuators 1 and 2 determines the wavelength dispersed by the prism that yields the strongest feedback. In this work, the micromirror is consistently resonant at $2.06 \mathrm{kHz}$ through a $10 \mathrm{~V}_{\mathrm{ac}}$ signal applied to actuator 4 .

The wavelength tuning achieved from this configuration of the laser whilst Q-switching is shown in Figure 4. The laser was optimally aligned at $0^{\circ}$ tilt of the micromirror and exhibited a peak wavelength of $1030.2 \mathrm{~nm}$, with a full width half maximum (FWHM) linewidth of $2.1 \mathrm{~nm}$. The end-pump optical power was adjusted for all subsequent measurements to give an average laser output power of $5 \mathrm{~mW}$, limited to prevent thermal damage to the micromirror surface. A $5 \mathrm{~V}_{\mathrm{dc}}$ signal applied to actuator 1 resulted in a tilt angle of $-0.28^{\circ}$ and a peak laser output wavelength of $1037.6 \mathrm{~nm}$, with a FWHM linewidth of $0.5 \mathrm{~nm}$. A $4 \mathrm{~V}_{\mathrm{dc}}$ signal applied to actuator 2 resulted in a tilt angle of $0.11^{\circ}$ and a peak laser output wavelength of $1022.2 \mathrm{~nm}$, with a FWHM linewidth of $0.8 \mathrm{~nm}$. Continuous tuning was observed in this $15.4 \mathrm{~nm}$ range. Q-switch pulse durations between $460 \mathrm{~ns}$ and $740 \mathrm{~ns}$ were observed at a repetition rate of $2.06 \mathrm{kHz}$ (one laser pulse per MOEMS movement cycle).

The dominant limitation of the laser system is the MOEMS optical coating performance. The light absorbed within the gold layer causes heating and,

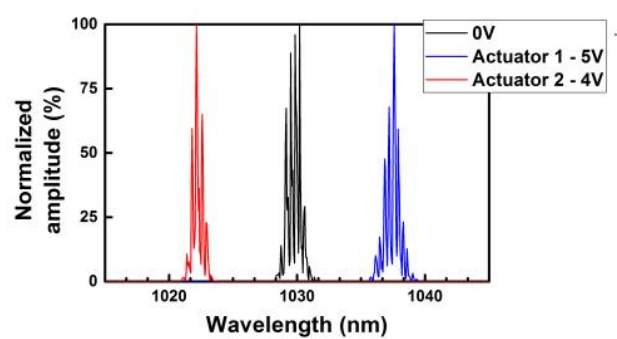

Figure 4: Optical spectra of the Q-switched laser at different fixed tilt angles

inherently, surface deformation. A low-stress, highreflectance dielectric coating will enable a constant micromirror surface curvature and average laser output powers in the Watt regime. Further improvement to laser performance will be made possible through changing the micromirror design. A 'straight line' resonant scan with no offset would enable shorter pulse durations and two laser pulses per MOEMS movement cycle.

\section{CONCLUSION}

Simultaneous Q-switching and wavelength tuning of a solid-state laser has been achieved for the first time through actuation of a single MOEMS mirror. A wavelength tuning range of $15.4 \mathrm{~nm}$ was achieved with laser pulse durations between $460 \mathrm{~ns}$ and $740 \mathrm{~ns}$ at a repetition rate of $2.06 \mathrm{kHz}$. With improvements to the MOEMS design and optical coating, such lasers could be power-scaled and made compatible with such applications as range finding, target tracking and optical gas sensing.

\section{REFERENCES}

[1] R. Bauer, A. Paterson, C. Clark, W. Lubeigt and D. Uttamchandani, "Output characteristics of Q-switched solidstate lasers using intracavity MEMS micromirrors," IEEE JSTQE, vol. 21, pp. 356-363, August 2014.

[2] R. Bauer, W. Lubeigt and D. Uttamchandani, "Dual Qswitched laser outputs from a single lasing medium using an intracavity MEMS micromirror array," Opt. Let., vol. 37, pp. 3567-3569, Sept 2012.

[3] V. Milanović et al., "Compact MEMS mirror based Q-switch module for pulse-on-demand laser range finders," SPIE MOEMS and Min. Sys. XIV, Feb 2015.

[4] M. Fabert, A. Desfarges-Berthelemot, V. Kermène and A. Crunteanu, "Temporal synchronization and spectral combining of pulses from fiber lasers Q-switched by independent MEMS micro-mirrors," Optics Express, Vol. 20, pp. 22895-22901, Sept 2012.

[5] A. Inoue, T. Komikado, K. Kinoshita, J. Hayashi and S. Umegaki, "Deformable mirror for mechanical Q-switching of laser-diode-pumped microchip laser," Jap. Jour. Appl. Phys., Vol. 46, pp. L1016-L1018, October 2007.

[6] A. Liu and M. Zhang, "A review of MEMS external-cavity tunable lasers," J. Micromech. And Microeng., Vol. 17, pp. R1-R13, January 2007.

[7] A. Paterson, R. Bauer, R. Li, C. Clark, W. Lubeigt and D. Uttamchandani, "Spectral and temporal control of Qswitched solid-state lasers using intracavity MEMS," SPIE Photonics West, July 2016. 